ameliorated Ang II-induced $\mathrm{Ca}^{2+}$ influx in human VSMC. In conclusion, TRPM2/NCX-induced increase in intracellular levels of calcium may be involved in hypertension-associated vascular dysfunction. Our data also suggests that oxidative stress regulates $\mathrm{Ca}^{2+}$ homeostasis through TRPM2-dependent mechanisms.

\section{CARDIAC DYSFUNCTION IN MICE WITH REDUCED STRIATIN EXPRESSION}

Kerry A Rostron*, Daniel N Meijles, Peter H Sugden, Angela Clerk. University of Reading

10.1136/heartjnl-2017-311726.159

Purpose Some protein kinases are regulated in STRIPAK complexes, with striatin (STRN) forming a scaffold. STRN mutations are associated with hypertension in humans and decreased expression of STRN causes arrhythmias and cardiomyopathy in dogs. Because striatin binds $\mathrm{Ca}^{2+}$-calmodulin, it was thought to participate in $\mathrm{Ca}^{2+}$-dependent signalling, but it is now recognised as regulatory $\mathrm{B}$ subunits of protein phosphatase PP2A. Thus, striatin holds the kinase in proximity to PP2A, maintaining it in an inactive state. Global striatin knockout is embryonic lethal in mice, but heterozygotes are viable and fertile. Our hypothesis is that heterozygote deletion of striatin will be detrimental to cardiac function in the context of hypertension.

Methods Cardiac function was assessed by echocardiography using a Vevo 2100 system. M-mode images of the short axis view were used for analysis of cardiac dimensions and ventricular function. Pulse-wave analysis of aortic flow was also performed. Following two baseline measurements, Strn \pm mice (10-12 weeks) were infused with the pro-hypertensive hormone angiotension II (AngII; $0.8 \mathrm{mg} / \mathrm{kg} / \mathrm{day} ; \mathrm{n}=5$ ) via osmotic minipumps for 24 hour. Cardiac function and aortic flow was measured and normalised to the mean of the baseline values.

Results Compared to baseline, heart rates were elevated by $18 \%$, whilst ejection fraction was reduced to $61 \%$ of baseline. Cardiac output was relatively preserved (87\% of baseline). The internal left ventricular (LV) diameter was increased to some extent, but systolic function was severely compromised. Posterior wall thickness during systole was reduced to $79 \%$ of baseline measurements and LV internal diameter was increased by $23 \%$ giving a calculated increase in systolic volume of $67 \%$.

Conclusions STRN plays an important role in maintaining systolic function during hypertension. Thus, the protein kinases that are regulated in striatin complexes must be significant regulators of cardiac contractility.

\section{BTK INHIBITORS: FRIENDS OR FOES?}

${ }^{1} \mathrm{D}$ Moreno-Martinez, ${ }^{1} \mathrm{~N}$ Binsaleh, ${ }^{1} \mathrm{~S}$ Daniels, ${ }^{1} \mathrm{~N}$ Dempsey-Hibbert, ${ }^{1} \mathrm{~S}$ Jones. ${ }^{1}$ Centre for Biomedicine Research, School of Healthcare Science, Manchester Metropolitan University, Chester Street, Manchester, M1 5GD

\subsection{6/heartjnl-2017-311726.160}

Introduction Bruton's tyrosine kinase (BTK) plays a crucial role in the development and maturation of B-cells. A common side effect of Ibrutinib, a BTK inhibitor approved for the treatment of chronic lymphocytic leukaemia (CLL), is active bleeding in the absence of vascular injury. The mechanisms by which ibrutinib alters haemostasis however are currently unclear. The aim of this study was to investigate the effects of ibrutinib on platelet and endothelial cell function in vitro, to determine the mechanisms that underpin ibrutinib-induced bleeding.

Methods Platelet rich plasma (PRP) collected from healthy volunteers was treated with increasing concentrations of ibrutinib for $15 \mathrm{~min}$ at $37^{\circ} \mathrm{C}$, prior to stimulation with collagen $(2 \mu \mathrm{g} /$ $\mathrm{ml})$ or $\operatorname{ADP}(10 \mu \mathrm{M})$. Platelet function and activation were measured by light transmission aggregometry (LTA) and flow cytometry respectively $\left(\mathrm{CD}_{2} \mathrm{P}^{+}, \mathrm{PAC}^{+}\right)$and platelet morphology analysed using scanning electron microscopy (SEM). Platelet signalling pathways were analysed by Western blotting and the generation of endothelial microvesicles (EMVs) from HUVECs enumerated by flow cytometry, following 24 hours treatment with ibrutinib (increasing concentrations from 0.1 $\mu \mathrm{M}$ to $10 \mu \mathrm{M})$.

Results Ibrutinib significantly reduced collagen-mediated platelet aggregation and activation in a dose-dependent manner $(\mathrm{p}<0.05)$. SEM analysis also demonstrated that collagen-mediated shape change and filopodia formation was defective following ibrutinib treatment. Consistent with these findings, signalling downstream of the collagen GPVI receptor was perturbed, with a marked reduction in $\mathrm{PLCI}^{3} 2$ phosphorylation. Ibrutinib only exerted mild inhibition of ADP-induced platelet aggregation $(p<0.05)$, which was accompanied by reduced $\mathrm{PLA}_{2}$ activation and inhibition of VASP dephosphorylation. Additionally, our results demonstrated that at low concentrations, ibrutinib reduced the generation of pro-thrombotic EMVs, an effect that is reversed at the highest concentrations $(\mathrm{p}<0.05)$.

Conclusion Ibrutinib reduces collagen and ADP-mediated platelet aggregation, and activation by reducing phosphorylation of $\mathrm{PLCI}^{3} 2$ and $\mathrm{PLA}_{2}$ and inhibiting VASP dephosphorylation. In addition, ibrutinib also appears to alter endothelial cell function by reducing EMV release. Understanding the mechanisms by which ibrutinib alters haemostasis may lead to the identification of novel antithrombotic targets.

\section{COMBINATORIAL ANALYSIS OF EXOME SEQUENCING DATA AND COPY NUMBER VARIANTS IN CONGENITAL HEART DISEASE PATIENTS}

${ }^{1}$ Elisavet Fotiou* ${ }^{2}$ Simon Williams, ${ }^{1}$ Bernard Keavney. ${ }^{1}$ University of Manchester; ${ }^{2}$ Manchester

\subsection{6/heartjnl-2017-311726.161}

Congenital heart disease (CHD) is the most common type of birth defect in humans. Most cases of CHD are sporadic with the specific interactions between genetic variants and environmental factors involved in their pathogenesis uncharacterised. Various whole exome sequencing studies have identified de novo mutations in different genes; however they have only explained a small percentage of CHD cases.Previous work from the group and others has identified chromosomal regions where rare copy number variants $(\mathrm{CNVs})$ were significantly enriched in CHD cases compared to controls. We hypothesise that utilising available $\mathrm{CNV}$ data to prioritise candidate regions within which we will interrogate exome sequencing 